\title{
Three Dimensional Printing of High Dielectric Capacitor Using Projection based Stereolithography Method
}

\author{
Yang Yang ${ }^{4,1,3 \#}$, Zeyu Chen ${ }^{2, \#}$, Xuan Song ${ }^{3, \#}$, Benpeng $\mathrm{Zhu}^{5}$, Tzung Hsiai ${ }^{1, *}$, Pin-I Wu ${ }^{3}$, Rui \\ Xiong $^{4}$, Jing Shi ${ }^{4}$, Yong Chen ${ }^{3, *}$, Qifa Zhou ${ }^{2, *}$, K. Kirk Shung ${ }^{2}$ \\ ${ }^{1}$ Department of Bioengineering and Division of Cardiology, University of California, Los Angeles, \\ 410 Westwood Plaza, CA 90095-1600 \\ ${ }^{2}$.Department of Biomedical Engineering, USC Viterbi School of Engineering, University of \\ Southern California. 3650 McClintock Ave, Los Angeles, CA 90089 \\ ${ }^{3}$ Epstein Department of Industrial and Systems Engineering, Viterbi School of Engineering, \\ University of Southern California, 3715 McClintock Ave, GER 201, Los Angeles, CA 90089-0193 \\ ${ }^{4}$ Key Laboratory of Artificial Micro- and Nano-structures of Ministry of Education and School of \\ Physics and Technology, Wuhan University, Wuhan 430072, P. R. China \\ ${ }^{5}$ School of Optical and Electronic Information, Huazhong University of Science and Technology. \\ Wuhan 430074, P.R.China \\ ${ }^{\#}$ These authors contributed equally to this work.
}

*Correspondence and requests for materials should be addressed to Y. Chen (email:yongchen @ usc.edu) and Q. Zhou (email: qifazhou@usc.edu).

\begin{abstract}
We report that efficient high dielectric polymer/ceramic composite materials can be optically printed into three-dimensional (3D) capacitor by the projection based stereolithography (SLA) method. Surface decoration of $\mathrm{Ag}$ on $\mathrm{Pb}(\mathrm{Zr}, \mathrm{Ti}) \mathrm{O}_{3}(\mathrm{PZT} @ \mathrm{Ag})$ particles were used as filler to enhance the dielectric permittivity. Polymer nanocomposites were fabricated by incorporating PZT@Ag particles into the photocurable polymer solutions, followed by exposure to the digitally controlled optical masks to generate 3D structures. The dielectric permittivity of Flex/PZT@Ag composite reaches as high as 120 at $100 \mathrm{~Hz}$ with $18 \mathrm{vol} \%$ filler, which is about 30 times higher than that of pure Flex. Furthermore, the dielectric loss is as low as 0.028 at $100 \mathrm{~Hz}$. The results are in good agreement with the effective medium theory (EMT) model. The calculated specific capacitance of our 3D printed capacitor is about $63 \mathrm{~F} \mathrm{~g}^{-1}$ at the current density of $0.5 \mathrm{~A} \mathrm{~g}^{-1}$. Cyclic voltammetry (CV) curves indicate $3 \mathrm{~d}$ printed capacitors possess low resistance and ideal capacitive properties. These results not only provide a tool to fabricate capacitor with complex
\end{abstract}


shapes but lay the groundwork for creating highly efficient polymer-based composites via 3D printing method for electronic applications.

Keywords: 3D printing, capacitor, high dielectric permittivity, photopolymerization, Comsol Multiphysics.

\section{Introduction:}

There is an enormous increase in demand for electric energy storage devices with high energy density and high power density due to the development of modern societies. Dielectric capacitors are capable of charging within milliseconds and have high fatigue and retention properties, making them an attractive method for electrical energy storage $e^{1-7}$. Recently, there has been increased attention on the production of high energy density capacitors through the use of high dielectric ceramics, high breakdown polymers, and composites of those materials ${ }^{8-11}$. Polymer/ferroelectric ceramic composites and polymer/conductive filler percolative dielectric composites are two types of materials that have been widely studied. There have been significant improvements in the development of such materials; however, the fabrication process have been restricted by the current technology (e.g. dropcasting, spin-coating, hot pressing and roll coating), especially for complicated structures ${ }^{12,13}$. Microfabrication of three-dimensional (3D) structures has drawn great research interests in materials science and engineering. This technology has found diverse applications in a wide variety of fields such as integrated electronics, tissue engineering and other advanced intelligent devices ${ }^{14-16}$. Some widely used 3D fabrication methods include lithography, direct ink printing, materials self-assembly and 3D printing ${ }^{17-20}$. Stereolithographic Apparatus (SLA) method is a 3D printing method that has been widely used to produce high-fidelity patterns and engineering products due to its low cost, efficiency and easy processability ${ }^{21}$. The general procedure of using SLA to build 3D structures involves the exposure of light to regions of photocurable liquid or polymer composite material to create cross-link in the regions. Once a single layer is formed with the light, the sample is translated to allow the next layer to be formed. The actual patterning can be programmed based on computer-aided design (CAD) models. In the present work, we introduce a new strategy to produce 3D structured capacitor based on high dielectric polymer/ceramic composites with Ag decorated PZT (denoted as PZT@Ag) as filler by the projection based SLA method. PZT@Ag hybrid particles were 
synthesized via a seed-mediated growing process by a redox reaction between silver nitrate and ethylene glycol. The surface of PZT@Ag nanoparticles were treated with 3-aminopropyltriethoxysilane to improve the compatibility and dielectric properties ${ }^{22,23}$. The material is based on the commercial photocurable resin and PZT@Ag to produce a range of high dielectric permittivity and high-fidelity patterns embedded as part of a 3D printed capacitor. We demonstrate that various 3D structures with excellent capacitive property can be made based on SLA method and have the potential usage in various applications such as printed circuit board, high-k gate dielectrics and embedded passive components.

\section{Experimental Section:}

Materials: PZT particles with an average diameter of $3 \mu \mathrm{m}$ (DeL. Piezo Specialties, LLC, West Palm Beach, FL), aqueous solution of $\mathrm{H}_{2} \mathrm{O}_{2}(30 \mathrm{wt} \%)$, toluene, ethylene glycol, silver nitrate (AgNO3), 3-aminopropyltriethoxysilane and other organic reagents or solvents were supplied by Sigma. PZT particles were ballmilling for 24 hours to get an average size of $400 \mathrm{~nm}$. Unless otherwise specified, all the chemicals were used as received. The Flex resin (Photocurable resin, Makerjuice Lab) is selected in our study due to its excellent photosensitivity. It contains 60-90wt\% Methacrylated monomer, 5-10wt $\%$ 1,6-Hexanediol methacrylate, 5-15wt\% Acrylated monomer and 0.1-1wt\% photoinitiator (Chemical structures are shown in Supporting information, Fig S1).

Hydroxylation of as received PZT nanoparticles: $10 \mathrm{~g}$ PZT particles were added into $50 \mathrm{ml}$ aqueous solution of $\mathrm{H}_{2} \mathrm{O}_{2}(30 \mathrm{wt} \%)$ in a round-bottomed flask. The mixture was sonicated for 30 min and then heated at $105^{\circ} \mathrm{C}$ for $4 \mathrm{~h}$. The nanoparticles were recovered by centrifugation. The obtained PZT particles were washed with deionized water for three times and then dried under vacuum at $80^{\circ} \mathrm{C}$ for $12 \mathrm{~h}$. The hydroxylated PZT particles were named as PZT-OH (Supporting information, Figure S2a).

Functionalization of PZT-OH by chemical groups: $10 \mathrm{~g}$ PZT-OH nanoparticles and $2 \mathrm{~g}$ (3-aminopropyl)triethoxysilane (APTES) were first added into $80 \mathrm{ml}$ toluene in a round-bottomed flask and sonicated for $30 \mathrm{~min}$, and then the mixture was heated at $100{ }^{\circ} \mathrm{C}$ for $2 \mathrm{~h}$. The nanoparticles were by centrifugation. The obtained nanoparticles were washed with toluene two times and were dried under vacuum at $80{ }^{\circ} \mathrm{C}$ for $12 \mathrm{~h}$ and named as PZT-SH. 
Grafting of nano-Ag particles onto the surface of PZT-SH: 10 g PZT-SH was added into 50 $\mathrm{ml}$ ethylene glycol in a flask and sonicated for $30 \mathrm{~min}$. Then ethylene glycol containing $0.5 \mathrm{~g}$ $\mathrm{AgNO}_{3}$ was slowly added. The mixture was heated to $160{ }^{\circ} \mathrm{C}$ and stirred under nitrogen for $2 \mathrm{~h}$. The particles were recovered by centrifugation and were washed with water and acetone several times. The obtained particles were denoted as PZT@Ag (Supporting information, Figure S2a).

Preparation of Flex/PZT@Ag composites: The printing method that was used is the same as the technology developed by Song ${ }^{22}$.PZT@Ag particles were mixed with Flex resins and put in the ultrasonic bath for $1 \mathrm{~h}$ and then stirred for 5 hours before use. Using a bottom-up projection based approach, a Mask Image Projection based Stereolithography (MIP-SLA) process by integrating ceramic tape-casting and a two-channel sliding design has been developed for the mixed slurry. Due to the added PZT@Ag particles, the mixed slurry has much higher viscosity and lower curing depth than the original photocurable resin. In our method, an integrated tape-casting system is used to recoat thick layers on a transparent glass substrate; uniform thin layers with smaller thickness are then recoated by moving the platform to form a desired gap between the previously cured layers and the glass substrate. After the layer recoating, mask images are projected upwards onto the bottom of the substrate. The newly cured layer is separated from the substrate using a two-channel sliding design based on controlled shearing force between the cured layer and the coated film on the glass substrate ${ }^{24}$.

Characterization: The microstructure of PZT, PZT@Ag particles and the fractured cross-surface morphology of the composites were examined by scanning electron microscopy (SEM, HITACHI S-4800) and transmission electron microscopy (TEM, JEM-2010). FTIR spectra of the carbon nanotube were collected by using FT/IR 420 Fourier Transform Infrared Spectrometer. Optical microscope images were taken on a TF probe microspectrophotometer SP100MS. The dielectric properties and AC conductivity were measured using a precise impedance analyzer (Agilent 4294A) at room temperature in the frequency range from $100 \mathrm{~Hz}$ to $100 \mathrm{MHz}$. For the dielectric test, hybrid films with sizes of $2 \times 2 \mathrm{~cm}$ and different filler loading were made by lighting for $10 \mathrm{~s}$. Au electrodes with a thickness of $200 \mathrm{~nm}$ were sputtered on both sides of the hybrid film before the measurement. Cyclic voltammetry and galvonostatic charge-discharge measurements of 3D printed capacitors were carried out on a supercapacitor test system (Solartron, 1470E) within the voltage range from 0 to $0.8 \mathrm{~V}$. The resolution of the DMD chip (Texas 
Instrument, Dallas, TX) is $1024 \times 768$, the output light intensity of the projector is $\sim 31.6 \mathrm{~W} / \mathrm{m}^{2}$.

\section{Results and Discussion:}

The scheme of the preparation process of PZT@Ag nanoassemblies (Supporting information, Fig S2a) will be discussed in the experimental section. TEM image reveals that PZT is uniformly coated with Ag particles (Supporting information, Fig S2c). The comparison of FTIR spectrum of PZT and PZT@Ag characterize the presence of (3-aminopropyl)triethoxysilane (APTES) on the surface of PZT@Ag (Supporting information, Fig S2d).

The challenge of the fabrication of polymer/nanoparticle composites is the reduction in cure depth by the incorporation of the filler. Due to the high refractive index of ceramic powders when compared to photocurable resin, the cure depth of the mixed ceramic slurry is significantly reduced. The relation between the cure depth and energy input is defined by the Jacob's equation as $^{25}: C_{d}=D_{P} \ln \left(\frac{E}{E_{c}}\right)$ where $C_{d}$ is the cure depth, $E$ is the energy density of incident light, $E c$ is the critical energy density, and $D_{p}$ is the resin sensitivity defined as: $D_{p}=\frac{2 d_{50}}{3 Q} \frac{n_{0}^{2}}{\Delta n^{2}}$, where $d_{50}$ is the average particle size; $\Delta n$ is the refractive index difference between the ceramic particle $\left(n_{p}\right)$ and the liquid resin $\left(n_{0}\right.$, i.e. $\left.\Delta n^{2}=\left(n_{p}-n_{0}\right)^{2}\right)$. The cure depth is related to the refractive index difference between the ceramic and the resin, the particle size, and the solid loading of ceramic powders in the suspension. The coating of Ag nanoparticles will lead to the increase in the refractive index of the particle and the further decrease in the cure depth, thus the filler loading will remain insufficiently high. More filler will lead to a smaller cure depth as an impediment to the fabrication process. The light intensity of our designed projection system is $31.6 \mathrm{~mW} / \mathrm{cm}^{2}$. The cure depth after projecting the mask image for 10 seconds is measured to be $40 \mu \mathrm{m}$ for Flex/PZT@Ag with 18vol\% filler.

The schematic diagram of the fabrication process of high dielectric material patterns by $3 \mathrm{D}$ printing technology is shown in Figure 1a. The 3D structure was fabricated in a bottom-up, additive fashion directly from digital designs layer by layer, without using the milling or molding 
processes. Thus complex objects can be produced directly from CAD models. First, mask images were projected upwards onto the bottom of the substrate to cure one layer of the composite. Then, a uniform thin layer was recoated by moving both the substrate in the $X$ axis and the platform in the $Z$ axis to form a desired gap between the previously cured layers and the glass substrate. After the projection of the mask image, a new layer would be formed. The thickness of one layer is controlled by moving the $Z$ stage to form a gap that is smaller than the cure depth related to the projection time and the mixture of the composite itself. For our composite, one layer thickness of $25 \mu \mathrm{m}$ was used for exposing mask images for $10 \mathrm{~s}$. The patterns of the 3D structure were controlled by the light exposure patterns . Different sizes of hexagonal patterns with a maximum edge width of $1 \mathrm{~mm}$ (Figure 1b.2) and minimum edge width of $200 \mu \mathrm{m}$ (Figure 1b.4) were fabricated. One layer $(20-40 \mu \mathrm{m})$ of pattern is flexible with potential applications in mechanical and electronic engineering. The optical microscopy images in Figure 1b.(3) \& (4) show that there are no cracks and the edges of the hexagonal patterns are smooth, which demonstrates the homogeneity of the mixed composites and the printing technology. Figure 1c.(1) shows 14 hexagonal bulges with an average diameter of $400 \mu \mathrm{m}$ and height of $600 \mu \mathrm{m}$ (about 24 layers), revealing the smooth surfaces for both the hexagonal bulges and holes. The results demonstrate good compatibility between the individual layers and the SLA technology for using polymer/ceramic composites to fabricate 3D structures for designed dielectric and electrical properties.

To further illustrate 3D fabrication of complicated objects, we built a star and a bowl using Flex and 18 vol\% PZT@Ag based on the 3D printing method (Figure 2). Different shapes of Flex/PZT@Ag nanocomposites can be designed and fabricated by 3D printing. A process control software system was developed by using Microsoft Visual C++. Figure 2 a.(1) and b.(1) illustrate the graphical user interface of the developed software system. The software system is capable of slicing a given CAD model and dynamically outputting mask images to the designed projection system. The resolution of the DMD chip is $1024 \times 768$. It is clear to see that the star and bowl models were fabricated layer-by-layer. Different layers of the star and bowl attach well with each other. There are no cracks between the layers, which are important for the electrical properties of the composites. The optical micrographs of different parts of the star and bowl show that the surface of each layer is smooth, which demonstrate that the PZT@Ag particles are 
homogeneously dispersed in Flex matrix. The homogeneous distribution is also critical for the fabrication and achieving desired electrical property of the composites. Figure 3 shows SEM images of the 3D printed hexagonal bulges patterns capacitor. The fracture surface of Pure Flex demonstrates that it is smooth due to the photocuring process. A comparison of the fracture surfaces of the composite of Flex/PZT@Ag (2vol\% and 10 vol\%) demonstrates that it is self-connected into a continuous network, which is attributable to chemical groups added on the surface of the filler that improves the compatibility of the ceramic particles with the polymer matrix. Homogenous filler distribution is important to achieve the improvement of dielectric properties and the capacitive behavior. When further increasing the volume fraction to $18 \mathrm{vol} \%$, some of the filler aggregates begin to form large clusters, and more layered swells and pores emerge on the cross surface (Figure 3d). This restricts us from further increasing the filler loading in the mixed slurry.

Figures 4 show the dependence of dielectric permittivity $(\varepsilon)$ and loss tangent $(\tan \delta)$ on frequency with different volume fractions in the frequency range of $100 \mathrm{~Hz}-100 \mathrm{MHz}$ at room temperature. It is found that both $\varepsilon$ and tan $\delta$ increase gradually with filler content. The hybrid film has the largest dielectric permittivity of $120(100 \mathrm{~Hz})$ when the content of PZT@ Ag is 18vol\%. Dielectric loss remains at a low level $(0.028$ at $100 \mathrm{~Hz})$ at the same loading (Figure $4 \mathrm{c})$. In comparison with pure Flex $(\varepsilon=4)$, the dielectric permittivity of the composite is nearly 30 times higher. It is also shown that the incorporation of conducting fillers on the surface of ceramic particles in the polymer matrix will result in the increase in the dielectric permittivity ${ }^{26-28}$. The significant enhancement of dielectric permittivity is attributed to the enhancement of Maxwell-Wagner-Sillars (MWs) effect ${ }^{29}$. That is, the increment of conductivity of the interlayer between PZT and Flex by Ag enhances the space charge polarization and MWs effect, which plays a very important role in improving the dielectric permittivity ${ }^{30,31}$. Lots of charges are blocked at the interfaces between the filler and polymer matrix, rendering a significant contribution to the increment of the dielectric permittivity ${ }^{32}$. In addition, a clear frequency dependence is observed that is attributed to the interfacial polarization or commonly referred to MWs-polarization of filler and polymer interface ${ }^{33}$. Another point that contributes to the improvement of dielectric property is that the effective poling of PZT and the improvement of the electrical distortion by the surface modification (Supporting information, Figure S3). 
To clarify the mechanisms of the composites' dielectric properties, the Logarithmic model, the Maxwell-Garnett (M-G) model, the effective medium theory (EMT) model, and the Burgeeman model were implemented to predict the dielectric permittivity of the composite at 100 $\mathrm{Hz}$ and room temperature (Figure $4 \mathrm{~b}$ ). These four models can be expressed as ${ }^{34-36}$

Logarithmic model: $\ln \varepsilon=(1-f) \ln \varepsilon_{1}+f \ln \varepsilon_{2}$

Maxwell-Garnett model: $\varepsilon=\varepsilon_{1}\left(1+\frac{3 f\left(\varepsilon_{2}-\varepsilon_{1}\right)}{2 \varepsilon_{1}+\varepsilon_{2}-f\left(\varepsilon_{2}-\varepsilon_{1}\right)}\right)$

EMT model: $\quad \varepsilon=\varepsilon_{1}\left[1+\frac{f\left(\varepsilon_{2}-\varepsilon_{1}\right)}{\varepsilon_{1}+n(1-f)\left(\varepsilon_{2}-\varepsilon_{1}\right)}\right]$

Burgeeman Model: $(1-f)\left(\frac{\varepsilon_{1}-\varepsilon}{\varepsilon_{1}+2 \varepsilon}\right)+f\left(\frac{\varepsilon_{2}-\varepsilon}{\varepsilon_{2}+2 \varepsilon}\right)=0$

where $\varepsilon, \varepsilon_{1}$ and $\varepsilon_{2}$ are the dielectric constants of the composites, Flex matrix and PZT ceramic powder, respectively, while $f$ is the volume fraction of the PZT ceramics, and $n$ is the correction factor to compensate for the shape of the fillers used in ceramic-polymer composites.

Figure $4 \mathrm{~b}$ shows that the Burggeman model is consistent with experimental values at $2 \mathrm{vol} \%$ and $5 \mathrm{vol} \%$ loadings, but deviates significantly when the content reaches $18 \mathrm{vol} \%$ as the particle size distribution in our study is wide (Supporting information, Figure S2b). The EMT model is in close agreement with experimental values since it calculates effective properties for media with located symmetric inclusions ${ }^{37}$. With the surface modification of chemical groups, the compatibility between polymer resin and ceramic particles is improved and a homogeneous distribution of particles is achieved (Supporting information, Figure S1e). M-G model predicts lower dielectric permittivity, which deviates from the experimental data. The M-G model suggests that filler particles should be non-interacting, and roughly spherical ${ }^{38}$. In practice, the interaction between fillers becomes apparent and should not be ignored when the loading increases. The logarithmic model deviates significantly with the experimental value as it is an empirical one and usually too simple to properly describe the composite's property.

The energy loss due to the consumption of a dielectric material can be determined by the following equation: $W=\pi \varepsilon \xi^{2} f \tan \delta$, where $\xi$ is the electric field strength and $f$ is the frequency. In order to reduce the energy loss, a low loss tangent is preferred, especially at a high 
frequency, in high-energy devices ${ }^{38}$. Figure $4 \mathrm{c}$ shows that the loss tangent increases with the filler loading but still remains at a low level. The loss tangent measured at a certain frequency includes polarization loss and conduction loss. The conduction loss is caused by the charge flow through the composites, which depends on the electrical conductivity of the composites. In order to ascertain the low value of the conduction loss, AC conductivity of pure Flex and Flex /PZT@Ag composites with different concentrations of filler are presented in Figure 4d. It can be observed that the value of conductivity slowly increases for Flex /PZT@Ag composites with an increase of filler content but the value still remains at a low level $\left(10^{-8}\right)$ for the composites with $18 \mathrm{vol} \%$. Thus the conduction loss is small and the polarization loss plays a dominant role in these composites. Unlike three phase ceramic particle/metal particle/polymer composites, the direct connection of conductive particles on the surface of PZT is prohibited by the ceramic and polymer resin. The discretely fixed Ag nanoparticles on PZT surface have no chance to aggregate in forming a conductive path. The controlled separation between Ag particles suppresses occurrence of the "percolation" in the composites, thus the charge transfer is restricted and lead to a low loss tangent ${ }^{39,40}$.

Au electrode were sputtered on both sides of different types of 3D printed Flex/PZT@Ag part to act as the top and bottom electrodes, respectively. The 3D printed capacitor can be made in this way. Figure 5a shows cyclic voltammetry (CV) curves of the 3D printed hexagonal bulges patterns capacitor, which exhibits nearly rectangular shapes at different scan rates from $5 \mathrm{mVs}^{-1}$ to $200 \mathrm{mVs}^{-1}$, indicating this kind of capacitor pocesses low resistance and ideal capacitive properties. Such an excellent capacitance behavior can be mainly attributed to electronic double-layer capacitance. To more directly probe the capacitive response of the 3D printed capacitor, Figure 5(b) displays the gavanostatic (GA) charge/discharge curves of the capacitor at different current densities. The discharge curves are almost symmetrical to the corresponding charge curves, indicating the pseudo-capacitive contribution along with the double layer capacitance. The specific capacitances of different types of 3D printed capacitor were calculated from their charge/discharge curves according to the following equation: $C_{s p}=\frac{I \times \Delta t}{\Delta V}$,

Where $C_{s p}$ is the specific capacitance, $I$ is the constant discharging current density, $t$ is the discharging time, and $V$ is the voltage window ${ }^{41,42}$. The calculated specific capacitance of our 3D 
printed capacitor is about $63 \mathrm{~F} \mathrm{~g}^{-1}$ at the current density of $0.5 \mathrm{~A} \mathrm{~g}^{-1}$. This is consistent with the capacitance value of $65 \mathrm{~F} \mathrm{~g}^{-1}$ obtained from $\mathrm{CV}$ testing at a scan rate of $20 \mathrm{mVs}^{-1}$, and is comparable to that of other functionalized or compact-designed supercapacitors reported in literature ${ }^{43,44}$. A comparison of the specific capacitances of different types of $3 \mathrm{D}$ printed capacitors indicates a highly repeatable printing capacitance behavior. A comparison of the charge-discharge behavior of 3D printed Flex/PZT and Flex/PZT@Ag capacitor with the same filler loading (18 vol\%) indicate that Flex/PZT@Ag capacitors have a better performance due to the improved electrical distortion and polarization as shown in Supporting information FigS3. Little degradation was observed for the 3d printed hexagonal bulges patterns Flex/PZT@Ag capacitor device after 1000 cycles, which demonstrate the excellent electrochemical stability of the 3D printed capacitors (Supporting information, Fig S4 b).

\section{Conclusion:}

We demonstrate here a novel tool for fabricating functional capacitors with complex three dimensional structures by $3 \mathrm{D}$ printing. The fabrication method relies on embedding high dielectric particles in a photocurable polymer resin, which enables the use of the projection based SLA process. By decorating the surface of PZT particles with Ag coating, the remarkable enhancement of dielectric permittivity is achieved by improvement of effective poling of PZT particle and the MWS effect. Moreover, the low loss $(0.028$ at $100 \mathrm{~Hz})$ achieved is attributed to the suppression in electric distortion and the blockage of charge transfer by the polymer resins. The calculated specific capacitance of our 3D printed capacitor is about $63 \mathrm{~F} \mathrm{~g}^{-1}$ at the current density of $0.5 \mathrm{~A}$ $\mathrm{g}^{-1}$. Cyclic voltammetry (CV) curves indicate this kind of 3D printed capacitor has low resistance and ideal capacitive properties. The capability of fabricating complex 3D structures with excellent capacitive behavior makes Flex/PZT@Ag capacitor attractive as a potential candidate for future 3D printing high charge-storage and embedded devices in the high energy density devices. These results not only provide a tool for fabricating capacitor with complex structures but lay the groundwork for creating highly efficient polymer-based composites using 3D printing for electronic industry.

\section{Acknowledgments:}

We thank the support from National Science Foundation (NSF) Grant-1335476 and National 
Institutes of Health (NIH) Grant P41-EB002182, and NIH 1R01HL118650.

\section{Appendix A. Supporting information}

Supplementary data associated with this article can be found in the online version.

\section{References:}

1. J.R. Miller, P. Simon, Science 321 (2008) 651-652.

2.J. M. Tarascon, M. Armand, Nature 414 (2001) 359-367.

3. X.L. Yu, C.Z. Zhan, R.T. Lv, Y. Bai, Y.X. Lin, Z.H. Huang, W.C. Shen, X.P. Qiu, F.Y. Kang, Nano Energy 15 (2015) 43-53.

4. N. Arun, A. Jain, V. Aravindan, S. Jayaraman, W.C. Ling, M.P. Srinivasan, S. Madhavi, Nano Energy 12 (2015), 19-75.

5.Z.Y. Xing, B. Wang, W.Y. Gao, C.Q. Pan, J.K. Halsted, E.S. Chong, J. Lu, X.F. Wang, W.Luo, C.H. Chang, Nano Energy 11 (2015) 600-610.

6. B.Song, L.Li, Z.Y. Lin, Z.K. Wu, K-S Moon, C.P. Wong, Nano Energy 16 (2015) 470-478.

7. Z.K. Wu, Z. Lin, L. Li, B. Song, K.S. Moon, S.L. Bai, C.P. Wong, Nano Energy 10 (2014) 222-228.

8. Z. Li, B. Song, Z. Wu, Z.Y. Lin, Y. Yao, K.S. Moon, C.P. Wong, Nano Energy 11 (2015) 711-718.

9. M. Rahimabady, K. Yao, S. Arabnejad, L. Lu, V. P. W. Shim, D. C. W. Chet, Appl. Phys. Lett.100 (2012) 252907.

10. J.Z. Chen, J.L. Xu, S. Zhou, N. Zhao, C.P. Wong, Nano Energy 15 (2015) 719-728.

11. X.N. Lu, L.Y. Li, B. Song, K.S. Moon, N.N. Hu, G.L. Liao, T.L. Shi, C.P. Wong, Nano Energy, 17 (2015) 160-170.

12. M. Mikolajek, A. Friederich, C. Kohler, M. Rosen, A. Rathjen, K. Kruger, J.R. Binder, Adv. Eng. Mater. 17 (2015) 1294-1301.

13. L.A.Ramajo, M.M. Reboredo, M.S. Castro, J. Mater. Sci. 42 (2007) 3685-3691.

14. Z. Qin, B.G. Compton, J.A. Lewis, M. J. Buehler, Nat. Commun. 6 (2015) 7038.

15. B.Z. Tian, J. Liu, T. Dvir, L. Jin, J.H. Tsui, Q. Qing, Z.G. Suo, R. Langer, D.S. Kohane, C.M. Lieber, Nat. Mater. 11 (2012) 986-994.

16. V. Mironov, G. Prestwich, G. Forgacs, J. Mater. Chem. 17 (2007) 2054-2060.

17. A.Kuzyk, R. Schreiber, H. Zhang, A.O. Govorov, T. Liedl, N. Liu, Nat. Mater. 13 (2014) 862-866.

18. C.T. Hong, Y.H. Kang, J. Ryu, S.Y. Cho, K.S. Jang, J. Mater. Chem. A 3 (2015) 21428-21433.

19. J. Hu, M.F. Yu, Science 329 (2010) 313-316.

20. G. Villar, A.D. Graham, H. Bayley, Science 340 (2013) 48-52.

21. K.K. Kim, W. Zhu, X. Qu, C. Aaronson, W.R. McCall, S.C. Chen, D.J. Sirbuly, ACS Nano. 8 (2014) 9799-9806.

22. Z.M. Dang, J.K. Yuan, S.H. Yao, R.J. Liao, Adv. Mater. 25 (2013) 6334-6365.

23. J.X. Lu, K.S. Moon, C.P. Wong, J. Mater. Chem. 18 (2008) 4821-4826.

24. X. Song, Y. Chen, T.W. Lee, S.H .Wu, L.X. Cheng, J. Manufactur. Proce. 20 (2015) 456-464.

25. F.P. Jacobs, D.T. Reid, Rapid prototyping \&manufacturing: Fundamentals of stereolithography, Society of Manufacturing Engineer, Dearborn, MI, 1992.

26. Y. Yang, H.L. Sun, D.Yin, Z. Lu, J. Wei, R. Xiong, J. Shi, Z.Y. Wang, Z.Y. Liu, Q.Q. Lei, J. 
Mater. Chem. A. 3(2015) 4916-4921.

27. K.Li, H.Wang, F. Xiang, W. Liu, H. Yang, Appl. Phys. Lett. 95 (2009) 202904.

28. L. Xie, X. Huang, B.W. Li, C. Zhi, T. Tanaka, P. Jiang, Phys. Chem. Chem. Phys. 15 (2013) 17560-17569.

29. C.W. Nan, Y. Shen, J. Ma, Annu. Rev. Mater. Res. 40 (2010) 131-151.

30. M. Panda, V. Srinivas, A.K. Thakur, Appl. Phys. Lett. 93 (2008) 242908.

31. X.Y. Huang, P. Jiang, L. Xie, Appl. Phys. Lett. 95 (2009) 242901.

32. Y. Yang, B.P. Zhu, Z.H. Lu, Z.Y. Wang, C.L. Fei, D. Yin, R. Xiong, J. Shi, Q.G. Chi, Q.Q. Lei, Appl. Phys. Lett.102 (2013) 042904.

33. Z.M. Dang, L. Wang, Y. Yin, Q. Zhang, Q.Q. Lei, Adv. Mater. 19 (2007) 852-857.

34. Y. Yang, Z.Y. Wang, Y. Ding, Z. Lu, H.L. Sun, Y. Li, J. Wei, R. Xiong, J. Shi, Z.Y. Liu, Q.Q. Lei, APL Mater. 1 (2013) 050701.

35. Y. Yang, H.L. Sun, B.P. Zhu, Z. Wang, J.H. Wei, R. Xiong, J. Shi, Z.Y. Liu, Q.Q. Lei, Appl. Phys. Lett.106 (2015), 012902.

36. Y. Yang, B.P. Zhu, D. Yin, J.H. Wei, Z.Y. Wang, R. Xiong, J. Shi, Z.Y. Liu, Q.Q.Lei, Nano Energy 17 (2015) 1-9.

37. S. Prakash, K.B.R. Varma, Compos. Sci. Technol. 67 (2007) 2363-2368.

38. Z.M. Dang, T. Zhou, S.H. Yao, J.K. Yuan, J.W. Zha, H.T. Song, J.Y. Li, Q. Chen, Adv. Mater. 21 (2009) 2077-2082.

39. X.Y. Huang, P.K.Jiang, Adv. Mater. 27 (2015) 546-554.

40. J.Y. Park, W. Lu, Appl. Phys. Lett. 91 (2007) 053113.

41. X.H. Lu , G.M. Wang, T. Zhai , M.H. Yu , S.L. Xie, Y.C. Ling, C.L. Liang, Y.X. Tong, Y. Li, Nano Lett. 12 (2012) 5376-5381.

42.G.M. Wang, X.H. Lu, Y.C. Ling, T. Zhai, H.Y. Wang, Y.X. Tong, Y. Li, ACS Nano 6 (2012) 10296-10302.

43.J.M. Shen, A.D. Liu, Y. Tu, G.S. Foo, C.B. Yeo, M.B.C. Park, R.R. Jiang, Y. Chen, Energy Environ. Sci. 4 (2011) 4220-4229.

44. Z.Q. Niu, W.Y. Zhou, J. Chen, G.X. Feng, H. Li, W.J. Ma, J.Z. Li, H.B. Dong, Y. Ren, D.A. Zhao, S.S. Xie, Energy Environ. Sci. 4 (2011) 1440-1446. 


\section{Figure Captions:}

Figure 1 | (a) Schematic diagram of the fabrication of high dielectric material patterns by 3D printing technology, DMD denotes Digital Micrometer Device; (b) (1) one layer hexagonal patterns of Flex/PZT@Ag (18 vol\%) composites with different sizes, (2) flexibility of the hexagonal pattern with one layer, (3)\&(4) optical microscopy images of the patterns with different size; (c) (1)\&(3) 3 dimensional hexagonal bulges patterns, (2)\&(4) 3 dimensional hexagonal hole patterns of Flex/PZT@Ag (18 vol\%) composites printed by SLA technology in relation to a quarter dollar for comparison.

Figure 2 | Images of the miniaturised structures. (a) 1) The graphical user interface of the developed process control software system to print the star structure (inset shows the star structure by SolidWork), 2) A photograph of star structure with a quarter of dollar shown alongside for scale.( the length of the star is $1 \mathrm{~cm}$, the layers are $40 \mu \mathrm{m}$ and there are 100 overlapping exposures in total, 3) and 4) optical microscopy images of different parts of the star, the images show that the print copes well with the severely overhanging chassis; (b) shows a model of bowl printed with $40 \mu \mathrm{m}$ layers and 100 overlapping exposures in total. 1) the graphical user interface of the developed process control software systen to print the bowl structure (inset shows the bowl structure by SolidWork), 2)shows a photograph of star structure in relation to a quarter of dollar alongside the scale(diameter is $1 \mathrm{~cm}$ and height $0.4 \mathrm{~mm}$ ), 3 ) and 4) shows the edges and bottoms of the bowl, respectively.

Figure 3 | SEM imgaes of Fracture surface of 3D printed hexagonal bulges patterns Flex/PZT@Ag capacitor with filler fraction of 0 vol\% (a); 2 vol\%(b); 10 vol\%(c) and 18 vol\% (d).

Figure 4 | (a) Dependence of dielectric permittivity on frequency of Flex/PZT@Ag hybrid films with different filler loading at room temperature; (b) Comparison of experimental and theoretical dielectric permittivities of Flex/PZT@Ag composites as a function of filler loading at $100 \mathrm{~Hz}$ and room temperature; (c) Dependence of loss tangent on frequency of the Flex/PZT@Ag hybrid films with different filler loading at room temperature; (d) Dependence of AC conductivity of Flex/PZT@Ag composites on frequency with different filler loading at room temperature.

Figure 5 | Electrochemical measurements for the as-prepared 3D printed capacitor (a) Cyclic voltammetry (CV) curves for 3D printed hexagonal bulges patterns Flex/PZT@Ag capacitor at different scan rates $\left(5,10,50,100,200 \mathrm{mVs}^{-1}\right)$, (b) charge-discharge curves of at different current densities $(0.5,1.0$, 
3.0, 5.0, $10 \mathrm{~A} \mathrm{~g}^{-1}$ ), (c) specific cdapacitances of different types of 3D printed capacitors, (d) comparison of charge-discharge curves of 3d printed Flex/PZT and Flex/PZT@Ag capacitor. 


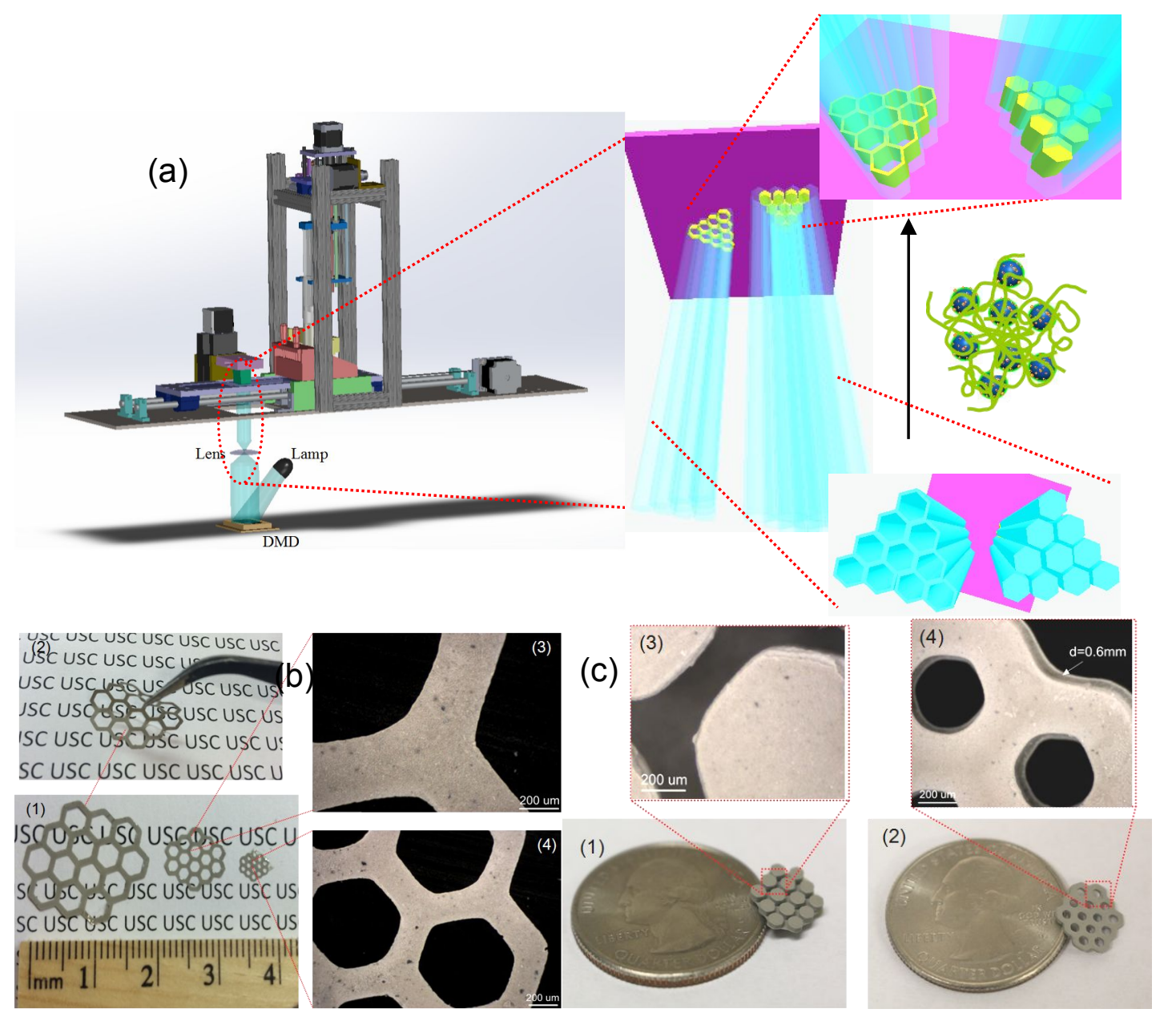

Figure 1 | (a) Schematic diagram of the fabrication of high dielectric material patterns by 3D printing technology, DMD denotes Digital Micrometer Device; (b) (1) one layer hexagonal patterns of Flex/PZT@Ag (18 vol\%) composites with different sizes, (2) flexibility of the hexagonal pattern with one layer, (3)\&(4) optical microscopy images of the patterns with different size; (c) (1)\&(3) 3 dimensional hexagonal bulges patterns, (2)\&(4) 3 dimensional hexagonal hole patterns of Flex/PZT@Ag (18 vol\%) composites printed by SLA technology in relation to a quarter dollar for comparison. 

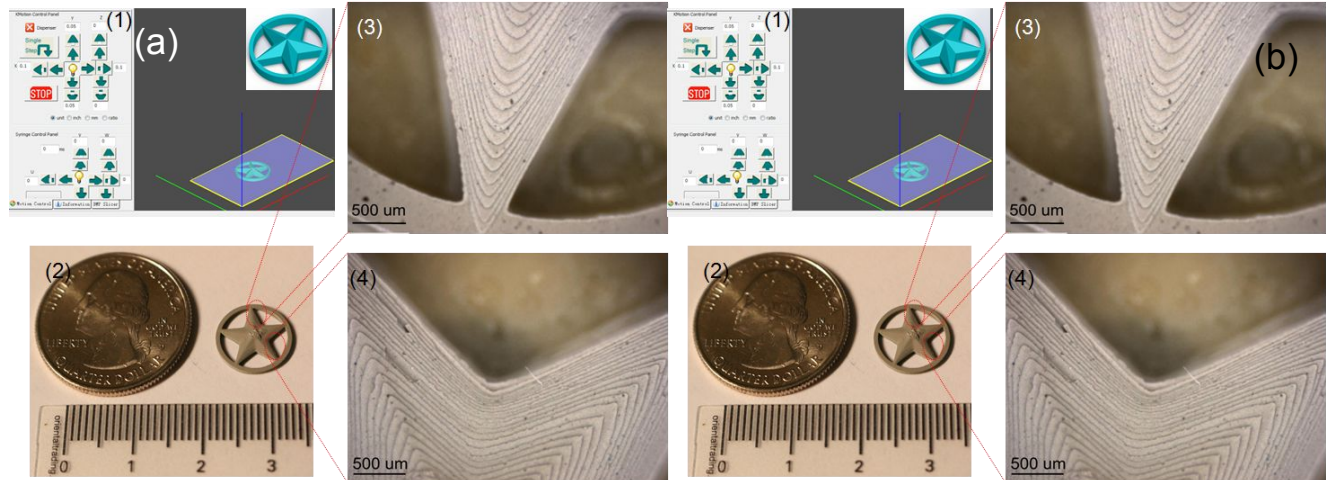

Figure 2 | Images of the miniaturised structures. (a) 1) The graphical user interface of the developed process control software system to print the star structure (inset shows the star structure by SolidWork), 2) A photograph of star structure with a quarter of dollar shown alongside for scale.( the length of the star is $1 \mathrm{~cm}$, the layers are $40 \mu \mathrm{m}$ and there are 100 overlapping exposures in total, 3) and 4) optical microscopy images of different parts of the star, the images show that the print copes well with the severely overhanging chassis; (b) shows a model of bowl printed with $40 \mu \mathrm{m}$ layers and 100 overlapping exposures in total. 1) the graphical user interface of the developed process control software systen to print the bowl structure (inset shows the bowl structure by SolidWork), 2)shows a photograph of star structure in relation to a quarter of dollar alongside the scale(diameter is $1 \mathrm{~cm}$ and height $0.4 \mathrm{~mm}$ ), 3 ) and 4) shows the edges and bottoms of the bowl, respectively. 

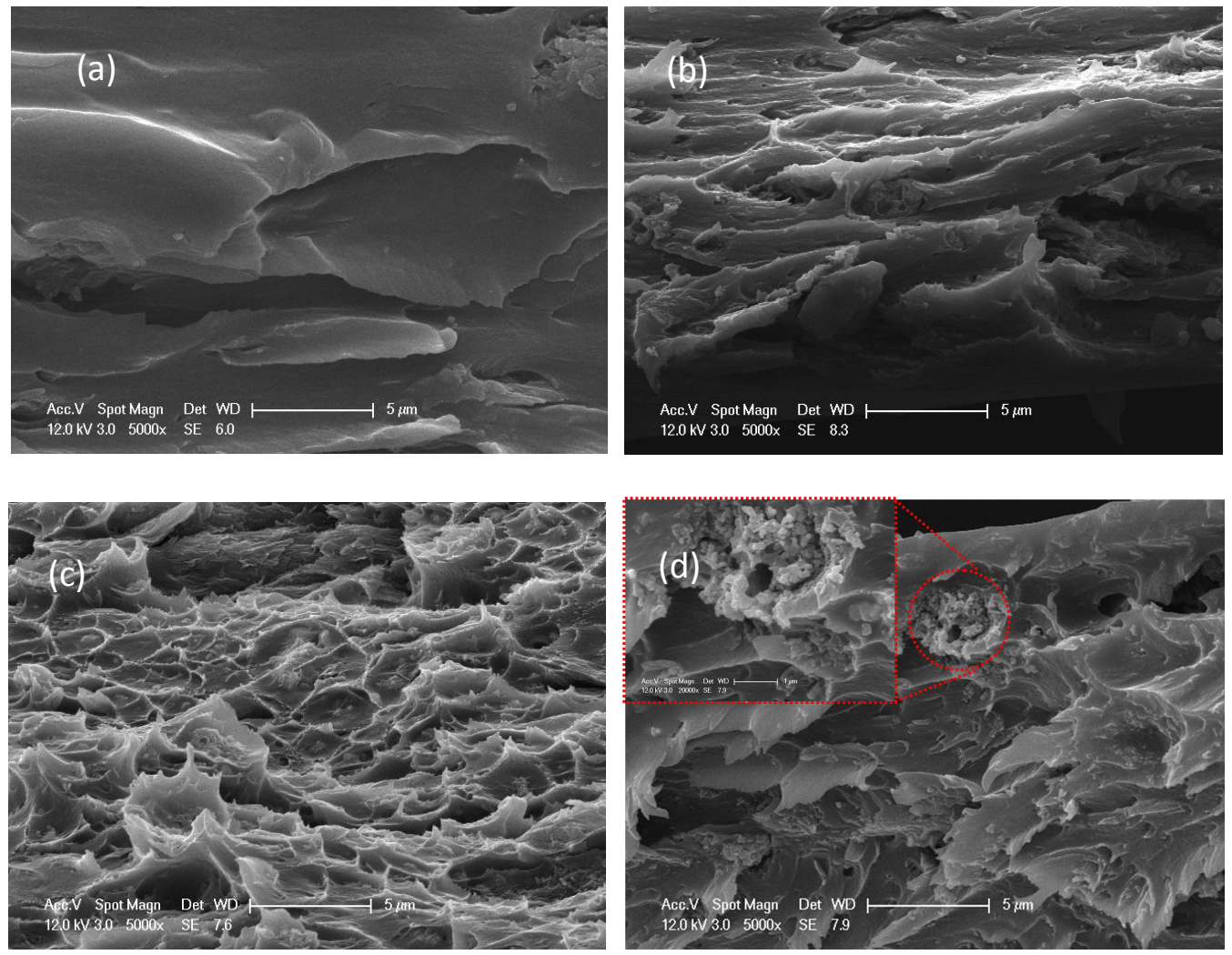

Figure 3 SEM imgaes of Fracture surface of 3D printed hexagonal bulges patterns Flex/PZT@Ag capacitor with filler fraction of 0 vol\% (a); 2 vol\%(b); 10 vol\%(c) and 18 vol\% (d). 

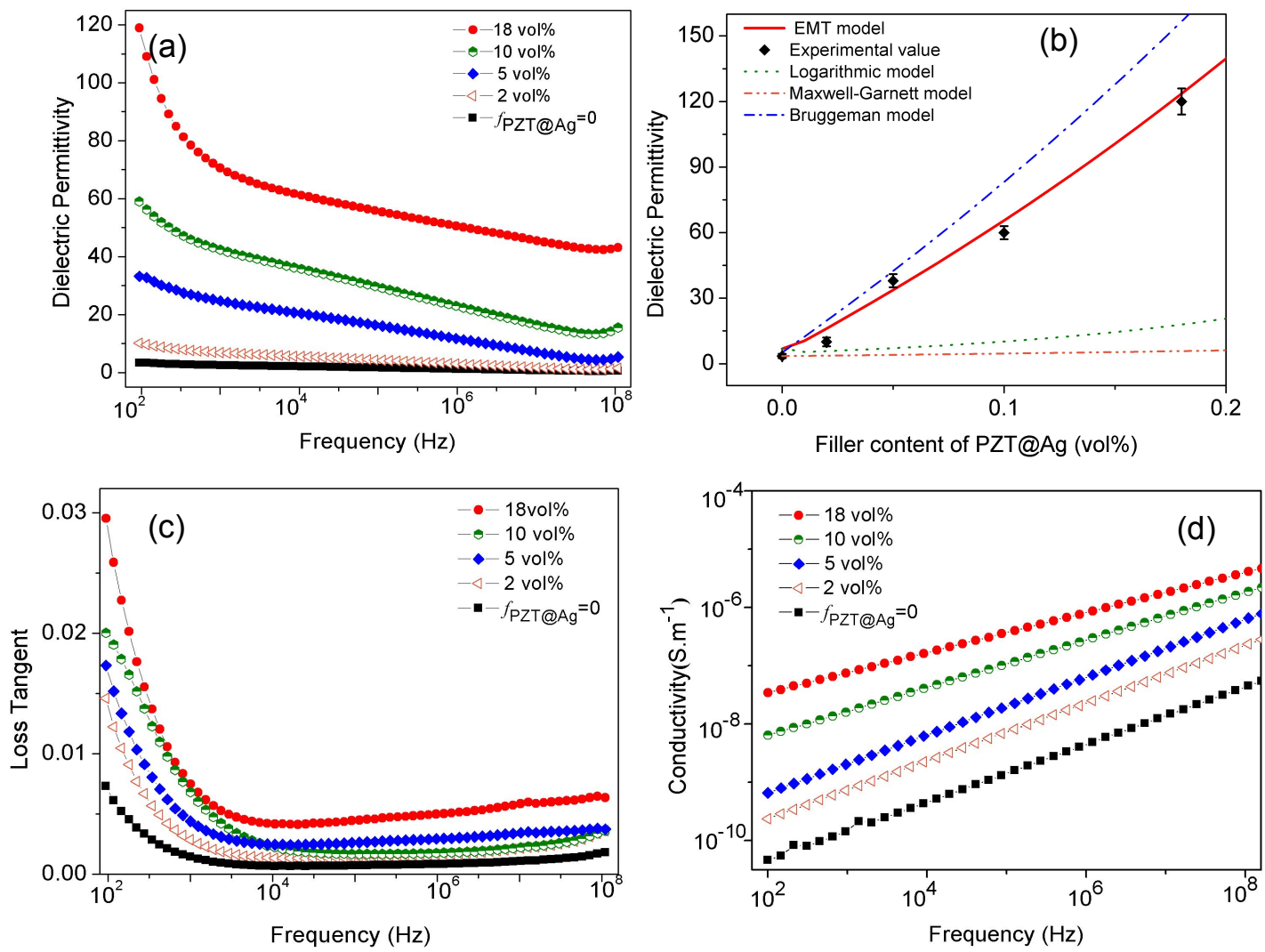

Figure 4 | (a) Dependence of dielectric permittivity on frequency of Flex/PZT@Ag hybrid films with different filler loading at room temperature; (b) Comparison of experimental and theoretical dielectric permittivities of Flex/PZT@Ag composites as a function of filler loading at $100 \mathrm{~Hz}$ and room temperature.

(c) Dependence of loss tangent on frequency of the Flex/PZT@Ag hybrid films with different filler loading at room temperature; (d) dependence of AC conductivity of Flex/PZT@Ag composites on frequency with different filler loading at room temperature. 

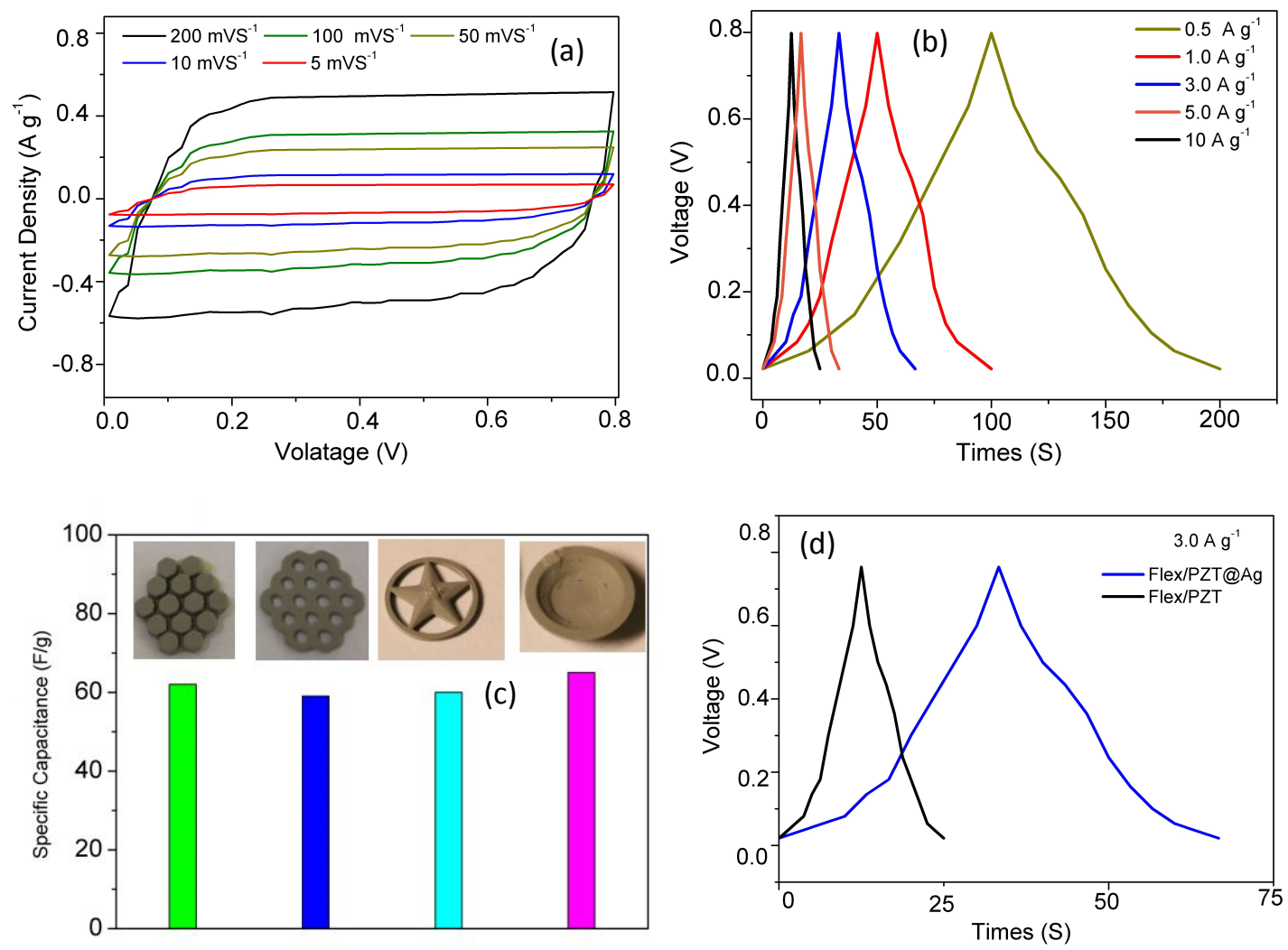

Figure 5. Electrochemical measurements for the as-prepared 3D printed capacitor (a) Cyclic voltammetry (CV) curves for 3D printed hexagonal bulges patterns Flex/PZT@Ag capacitor at different scan rates $\left(5,10,50,100,200 \mathrm{mVS}^{-1}\right)$, (b) charge-discharge curves of 3D printed hexagonal bulges patterns Flex/PZT@Ag capacitor at different current densities $\left(0.5,1.0,3.0,5.0,10 \mathrm{~A} \mathrm{~g}^{-1}\right)$, (c) specific cdapacitances of different types of 3D printed capacitors, (d) comparison of charge-discharge curves of 3d printed hexagonal bulges patterns Flex/PZT and Flex/PZT@Ag capacitor. 

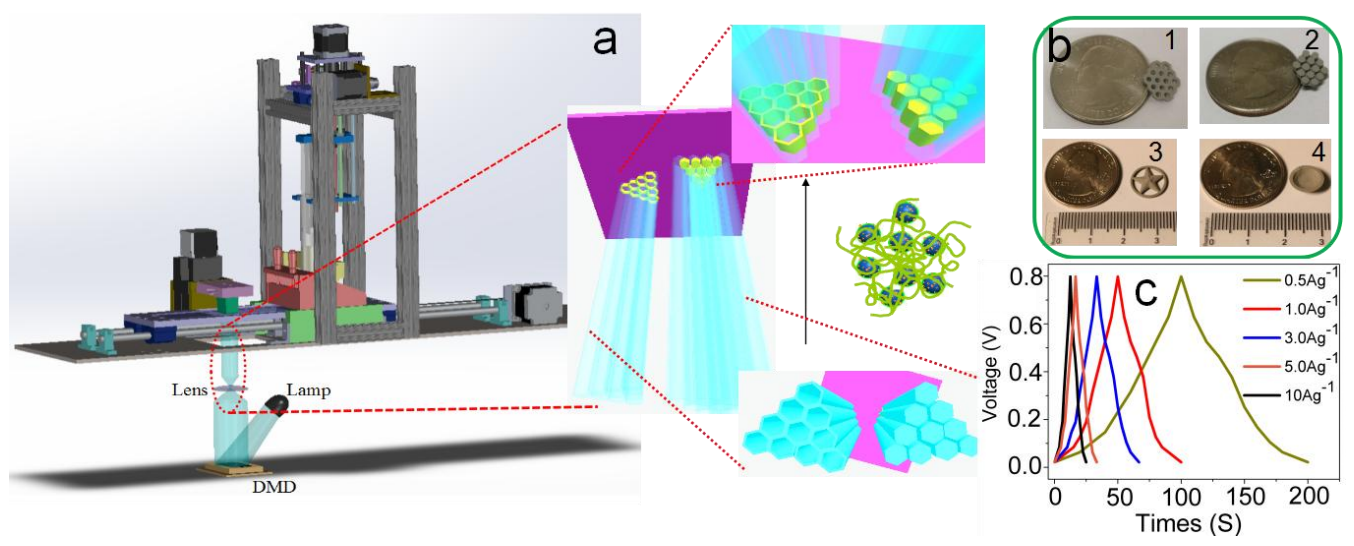

\section{Graphical Abstract:}

High dielectric polymer/ceramic composite materials can be optically printed into different types of three-dimensional (3D) capacitor (b1-b4) by the projection based stereolithography (SLA) method. Polymer nanocomposites were fabricated by incorporating PZT@Ag particles (Surface decoration of $\mathrm{Ag}$ on $\mathrm{Pb}(\mathrm{Zr}, \mathrm{Ti}) \mathrm{O}_{3}$ ) into photocurable polymer solutions, followed by exposure to digitally controlled optical masks to generate 3D structures (a). Charge-discharge curves (c) indicate $3 \mathrm{~d}$ printed capacitors possess low resistance and ideal capacitive properties. These results not only provide a tool to fabricate capacitor with complex shapes but lay the groundwork for creating highly efficient polymer-based composites with complicated structures via $3 \mathrm{D}$ printing method for electronic applications. 